\title{
Differences of intraoperative outcomes and postoperative complications between intrahepatic cholangiocarcinoma and colorectal liver metastasis in different surgical methods
}

\author{
Qichen Chen", Yiling Zheng", Jinghua Chen", Jianguo Zhou, Jianjun Zhao, Xinyu Bi, Zhiyu Li, \\ Zhen Huang, Yefan Zhang, Jianxiong Wu, Liming Wang, Hong Zhao^, Jianqiang Cai \\ Department of Hepatobiliary Surgery, National Cancer Center/National Clinical Research Center for Cancer/Cancer Hospital, Chinese Academy of \\ Medical Sciences and Peking Union Medical College, Beijing, China \\ Contributions: (I) Conception and design: Q Chen, Y Zheng, J Chen; (II) Administrative support: J Wu, L Wang, H Zhao, J Cai; (III) Provision of \\ study materials or patients: J Zhou, J Zhao, X Bi, Z Li, Z Huang, Y Zhang; (IV) Collection and assembly of data: Q Chen, Y Zheng; (V) Data analysis \\ and interpretation: J Chen; (VI) Manuscript writing: All authors; (VII) Final approval of manuscript: All authors. \\ \#These authors contributed equally to this work. \\ Correspondence to: Liming Wang; Hong Zhao. No. 17, Panjiayuan South Lane, Chaoyang District, Beijing, China. \\ Email: stewen_wang@sina.com; pumczhaohong@126.com.
}

Background: Hepatectomy is the only potentially curable treatment for intrahepatic cholangiocarcinoma (IHCC) and colorectal liver metastasis (CRLM). This study aimed to explore the difference in intraoperative outcomes and postoperative complications between IHCC and CRLM in different surgical methods including major hepatectomy and minor hepatectomy.

Methods: We included 319 patients with IHCC or CRLM who underwent hepatectomy at our hospital. According to major hepatectomy and minor hepatectomy, eligible patients were divided into two groups. In each group, the clinicopathological characteristics of IHCC and CRLM patients were compared, then propensity score matching (PSM) was performed based on the results. Intraoperative outcomes and postoperative complications were compared between IHCC and CRLM before and after PSM. Intraoperative variables, including intraoperative blood transfusion, duration of operation, and intraoperative blood loss, were used to evaluate the intraoperative conditions of patients. The postoperative complications were measured according to the Clavien-Dindo classification. Grade III to V complications were defined as major complications.

Results: The major hepatectomy group included 118 patients with IHCC and 93 patients with CRLM. IHCC patients presented a longer operation time and a higher postoperative complication rate than CRLM patients. The infection-related complication rate of the CRLM patients was significantly higher than the IHCC patients. In multivariate analysis, major hepatectomy for IHCC was independently associated with the presence of postoperative complications. The minor hepatectomy group included 146 IHCC patients and 62 CRLM patients. Compared with CRLM patients, IHCC patients presented a longer operation time. There was no significant difference in the intra-operative blood loss, postoperative complication rate, the major complications rate, and the minor complications rate between the IHCC patients and CRLM patients. Conclusions: This study revealed major hepatectomy for IHCC led to significantly higher morbidity of postoperative complications than CRLM patients. For minor hepatectomy, there was no difference in postoperative complications between IHCC and CRLM. More attention should be paid to improving the preoperative planning and surgical management of hepatic malignancies especially in the setting of IHCC.

Keywords: Intrahepatic cholangiocarcinoma (IHCC); colorectal liver metastasis (CRLM); hepatectomy; postoperative complication

^ ORCID: 0000-0002-6063-2099. 
Submitted Mar 30, 2021. Accepted for publication Jul 23, 2021.

doi: $10.21037 /$ tcr-21-553

View this article at: https://dx.doi.org/10.21037/tcr-21-553

\section{Introduction}

Nowadays, surgery is the only potentially curable treatment for common hepatic malignancies, including intrahepatic cholangiocarcinoma (IHCC) (1) and colorectal liver metastasis (CRLM) (2). For IHCC, radical surgery including hepatectomy and lymph node dissection only achieved a 5 -year survival rate of $30 \%$ (3). For CRLM, all patients with curable potentials were recommended to receive surgical treatment. The 5 -year survival rate after hepatectomy ranged from $20 \%$ to $45 \%(4,5)$. The basic condition for radical resection is to achieve $\mathrm{R} 0$ resection (6-9), which requires extended and complex hepatectomies (10). Hepatectomy is mainly divided into major and minor hepatectomy according to the extent of hepatectomy. Resection of three or more liver segments is defined major hepatectomy, which may facilitate the radical resection. However, perioperative morbidity and mortality may be higher than minor resection because of the wider surgical margin and removal of portal tributaries (11).

Patients with heterogeneous CRLM need to receive primary lesion radical resection firstly, hence when it came to second surgery for liver metastasis, abdominal adhesions might increase the probability of postoperative complications (12). Operations for different types of hepatic malignancy have different risk factors for postoperative complications. The most serious postoperative complication is liver failure caused by preoperative damaged liver function and cirrhosis, especially for IHCC patients (7). The increasing rate of postoperative complications leads to prolonged postoperative recovery time, increasing economic burden, and worse long-term prognosis of patients (13).

The preoperative liver function status and expected outcomes after hepatectomy for IHCC or CRLM were similar, but the postoperative mortality and morbidity rates were different. Compared with CRLM, IHCC presented worse mortality (0-2\% vs. 6-10\%) and morbidity (16-32\% vs. 48-50\%) (14-17). The differences under the same conditions of hepatectomy may be due to the specific characteristics of diseases and surgical management. Zhang et al. (15) found that major hepatectomy for IHCC was not associated with an overall survival benefit, yet was associated with increased perioperative morbidity. For CRLM, more and more researchers recommended promoting parenchyma-sparing hepatectomy $(18,19)$. Because it substantially decreased intraoperative blood loss and postoperative liver failure (20). Doussot et al. (10) suggested that IHCC patients were at higher risk inherently after major hepatectomy compared with CRLM patients. One possible reason was the higher frequency of portal lymphadenectomy for IHCC. Vascular and biliary injury are difficult to avoid and may result in some complications such as, lymphatic leakage, biliary ischemia, or bleeding. Better perioperative and operative management can translate into reducing mortality and morbidity. A better understanding of these differences may help improve perioperative management and thus patient outcomes, but no comprehensive comparative studies have been conducted.

The above observations motivated the current study. This study was conducted to explore the differences in intraoperative outcomes and postoperative complications between IHCC and CRLM in different surgical methods. The propensity score matching (PSM) was used to eliminate the significant differences in preoperative, intraoperative, and postoperative data. We present the following article in accordance with the STROBE reporting checklist (available at https://dx.doi.org/10.21037/tcr-21-553).

\section{Methods}

\section{Population}

Patients who underwent hepatectomy for IHCC or CRLM at Cancer Hospital, Chinese Academy of Medical Sciences from April 2011 and December 2018 were included. Inclusion criteria were: (I) Pathologically proven IHCC or CRLM. (II) Hepatic resection for curative intent. (III) Metachronous liver metastasis in CRLM patients; Exclusion criteria were: (I) Palliative-intent resection; (II) CRLM patients without having received primary tumor resection previously; (III) With other malignancies. The extent of hepatectomy included major and minor hepatectomy. Major hepatectomy was defined as the resection of more than two liver couinaud segments. The study was conducted in accordance with the Declaration of Helsinki (as revised in 2013). The study was approved by the Institutional Ethics Committee of Cancer Hospital Chinese Academy of 
Medical Sciences (ID NCC2019C-016). Informed consent was taken from all the patients.

\section{Data collection}

This was a retrospective study based on the CRLM and HICC datasets. Clinical variables included age, gender, American Society of Anesthesiology (ASA) score, body Mass Index (BMI), and preoperative test markers. Tumor-related variables included tumor size and number. Eligible patients were divided into two groups according to major hepatectomy and minor hepatectomy. The clinical covariates of patients with IHCC and CRLM were compared between the two groups to find differences between the two groups. In each group, PSM was performed based on the results. The primary outcomes included intraoperative variables and postoperative complications. The primary objective of this study was to compare the differences in intraoperative variables and postoperative complications between IHCC and CRLM patients, with a secondary objective to identify independent risk factors for postoperative complications in these patients.

Compared with CRLM patients, IHCC patients received significantly more frequent lymphadenectomy $(76.3 \% \mathrm{vs}$. $0.0 \%, \mathrm{P}<0.001)$ and less preoperative treatment $(7.6 \%$ vs. $67.7 \%, \mathrm{P}<0.001)$. Based on the significant distribution difference of preoperative treatment and lymphadenectomy (PTL) between those patients. The combination of the PTL on these two factors was constructed to eliminate the influence of the two on the outcomes and ensure the feasibility of PSM. The PTL was scored as 0 (nonpreoperative treatment with non-lymphadenectomy), 2 (preoperative treatment with lymphadenectomy), and 1 (all other combinations). Intraoperative variables, including intraoperative blood transfusion, duration of operation, and intraoperative blood loss, were used to evaluate the intraoperative conditions of patients. The postoperative complications were measured according to the ClavienDindo classification. Grade III to V complications were defined as major complications. If patients experienced multiple postoperative complications, the highest grade was used. The postoperative complications included general complications and surgical-related complications. Death or complications within 30 days after surgery are considered postoperative mortality and morbidity.

\section{Statistical analysis}

The Mann-Whitney $U$ test was performed to analyze continuous variables, including operation time and intraoperative blood loss, etc. The Chi-square or Fisher's exact test was used to analyze categorical variables. The independent predictive factors of postoperative complications were identified using univariable and multivariable logistic regressions. For patients undergoing the same type of hepatectomy, the PSM method was used to balance the imbalanced clinicopathological characteristics between IHCC and CRLM patients. In patients receiving major hepatectomy, age, preoperative serum gamma glutamyl transpeptidase (GGT) level, the diameter of the largest tumor, and tumor number were balanced. In patients receiving minor hepatectomy, ASA, the diameter of the largest tumor and PTL were balanced. Patients with ICHCC were matched in a 1:1 ratio to patients with CRLM, with a standard caliper width of 0.2. A two-tailed P value of less than 0.05 was considered significant. The SPSS version 22.0 software (Armonk NV, USA) and R software (http://www.r-project.org, 3.6.3) were used to perform the statistical analyses.

\section{Results}

In this study, 319 patients were included, including 164 IHCC patients and 155 CRLM patients. There were 118 IHCC patients and 93 CRLM patients undergoing major hepatectomy, respectively. Forty-six IHCC patients and 62 CRLM patients receiving minor hepatectomy, respectively. The outcomes were compared between HICC and CRLM patients in each group.

\section{Patients receiving major hepatectomy}

\section{Clinicopathological characteristics}

IHCC patients were significantly older $(\mathrm{P}=0.008)$ and presented with higher preoperative serum GGT levels $(\mathrm{P}=0.011)$. There was no significant difference in preoperative serum TBIL, AST, ALT, and ALB levels between the two gruops. IHCC patients presented significantly larger tumor size $(\mathrm{P}<0.001)$ and less multiple tumors $(\mathrm{P}<0.001$; Table 1$)$. IHCC patients received significantly more often lymphadenectomy $(76.3 \%$ vs. $0.0 \%$, $\mathrm{P}<0.001)$ and less preoperative treatment $(7.6 \%$ vs. $67.7 \%$, $\mathrm{P}<0.001)$. According to $\mathrm{PTL}$, there was no significant difference between IHCC and CRLM patients $(\mathrm{P}=0.095)$.

\section{Outcomes before and after PSM}

The median of operation time and intraoperative blood 
Table 1 Clinicopathological characteristics in patients receiving major liver resection before PSM

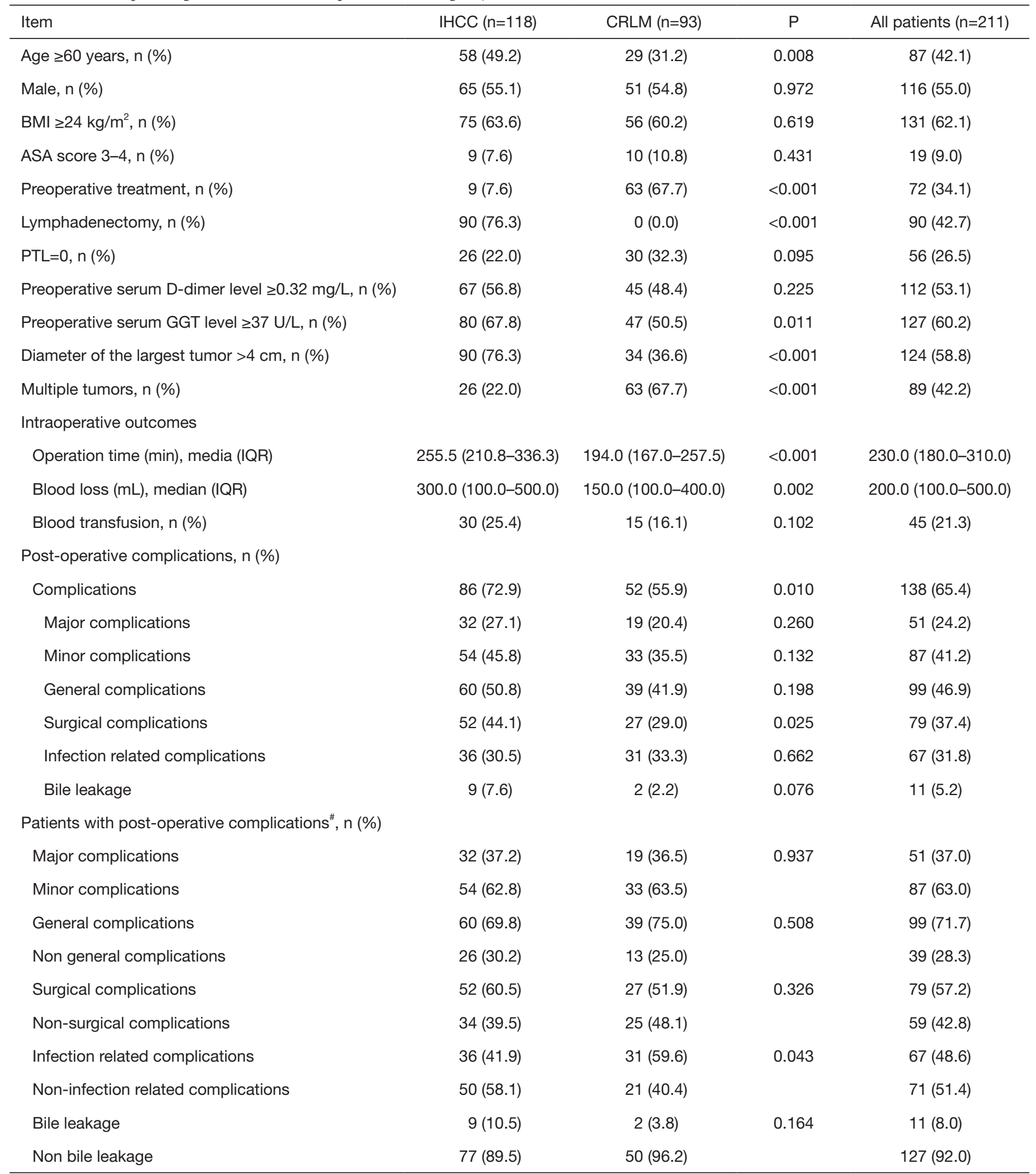

\#, IHCC $(n=86)$, CRLM ( $n=52)$, all patients $(n=138)$. PSM, propensity score matching; BMI, body mass index; ASA, American Society of Anesthesiology; PTL, pre-operative treatment and lymphadenectomy; GGT, gamma glutamyl transpeptidase. 
loss was 230.0 (IQR: 180.0-310.0) min and 200.0 (IQR: $100.0-500.0) \mathrm{mL}$, respectively. There were $21.3 \%$ of patients who received intraoperative blood transfusion. There was no postoperative mortality. And $65.4 \%$ of patients underwent postoperative complications (general complications: 99 patients, surgery-related complications: 79 patients, infection-related complications: 67 patients, bile leakage: 11 patients), including 51 with major complications and 87 with minor complications. IHCC patients presented longer operation time [Median (IQR): 255.5 (210.8-336.3) vs. 194.0 (167.0-257.5) min, $\mathrm{P}<0.001]$ and more intraoperative blood loss [Median (IQR): 300.0 (100.0-500.0) vs. $150.0(100.0-400.0) \mathrm{mL}$, $\mathrm{P}=0.002]$. The postoperative complication rate $(\mathrm{P}=0.010)$ and the surgical complications rate $(\mathrm{P}=0.025)$ were significantly higher in IHCC patients than in CRLM patients. In patients with postoperative complications, the infection-related complication rate was significantly higher in CRLM patients than in IHCC patients ( $\mathrm{P}=0.043$; Table 1).

Univariate analysis indicated that PTL $=2(\mathrm{P}=0.004)$, preoperative serum GGT level $\geq 37.0 \mathrm{U} / \mathrm{L}(\mathrm{P}=0.020)$, major hepatectomy for IHCC $(\mathrm{P}=0.011)$, operation time $(\mathrm{P}<0.001)$, and intraoperative blood transfusion $(\mathrm{P}=0.023)$ were significantly associated with postoperative complications. All the predictors $(\mathrm{P}<0.1)$ were included in the multivariate analysis, and it indicated that major hepatectomy for IHCC (OR 1.921, 95\% CI: 1.066-3.459, $\mathrm{P}=0.030)$ and PTL $=2($ OR $2.207,95 \%$ CI: 1.209-4.028, P=0.010) were significantly associated with higher morbidity of postoperative complications (Table 2).

After 1:1 PSM (Table 3), 46 IHCC patients and 46 CRLM patients undergoing major hepatectomy were matched successfully. Compared with CRLM patients, IHCC patients presented the longer operation time [Median (IQR): 241.5 (214.5-355.5) vs. 182.0 (148.8-250.0) min, $\mathrm{P}<0.001]$. The postoperative complication rate was significantly higher in the IHCC patients than in the CRLM patients $(\mathrm{P}=0.029)$, while the incidence of surgical complications was not significantly different $(\mathrm{P}=0.277)$. In patients with postoperative complications, the infectionrelated complication rate was more common in the CRLM patients ( $\mathrm{n}=18,72.0 \%)$ than in the IHCC patients $(\mathrm{n}=13,37.1 \%, \mathrm{P}=0.008$; Table 3).

In the univariate analysis listed in Table 2, major hepatectomy for IHCC $(\mathrm{P}=0.031)$, operation time $(\mathrm{P}=0.002)$ and PTL $(\mathrm{P}=0.021)$ were significantly associated with postoperative complications. The intraoperative blood loss tended to the presence of postoperative complications $(\mathrm{P}=0.062)$. In the multivariate analysis, major hepatectomy for IHCC (OR 2.673, 95\% CI: 1.095-6.521, P=0.031) was independently associated with higher morbidity of postoperative complications (Table 2).

\section{Patients receiving minor hepatectomy}

\section{Clinicopathological characteristics}

Over the study period, 46 IHCC patients and 62 CRLM patients who underwent minor hepatectomy were included. Compared to the CRLM patients, the IHCC patients presented larger tumor size $(45.7 \%$ vs. $15.5 \%, \mathrm{P}<0.001)$ but have no significant difference in the multiple tumors (8.7\% vs. $12.9 \%, \mathrm{P}=0.491)$. PTL $=1$ was more observed in the IHCC patients than CRLM patients (47.8\% vs. $22.6 \%$, $\mathrm{P}=0.006$; Table 4).

\section{Outcomes before and after PSM}

The median of operation time and intraoperative blood loss was 150.0(IQR:120.0-210.3) $\mathrm{min}$ and 200.0 (IQR:100.0-300.0) mL, respectively. And 2.8\% of patients received intraoperative blood transfusion. There was no postoperative mortality. In this study, $31.5 \%$ of patients underwent postoperative complications (general complications: 29 patients, surgery-related complications: 12 patients, infection-related complications: 20 patients, bile leakage: 2 patients), including 12 major complications and 22 minor complications. IHCC patients presented the longer operation time [Median (IQR): 203.0 (139.8-225.0) vs. 132.5 (108.3-172.0) $\mathrm{min}, \mathrm{P}<0.001]$, more intraoperative blood loss [Median (IQR): 200.0 (100.0-300.0) vs. 150.0 (95.0-200.0) $\mathrm{mL}, \mathrm{P}<0.001]$ and more intraoperative blood transfusion $(6.5 \%$ vs. $0.0 \%, \mathrm{P}=0.041$; Table 4$)$.

After 1:1 PSM, 31 IHCC patients and 31 CRLM patients undergoing minor hepatectomy were included in the study. Compared with CRLM patients, IHCC patients presented the longer operation time [Median (IQR): 187.0 (140.0-225.0) vs. 143.0 (109.0-171.0) min, $\mathrm{P}=0.013]$. There was no significant difference in the intraoperative blood loss [Median (IQR): 200.0 (100.0 300.0) vs. $200.0(100.0-200.0) \mathrm{mL}, \mathrm{P}=0.638$ ], postoperative complication rate $(\mathrm{P}=0.421)$, the major complications rate $(\mathrm{P}=0.688)$, the minor complications rate $(\mathrm{P}=0.544)$, the surgical complications rate $(\mathrm{P}=0.718)$ and the general complications rate $(\mathrm{P}=0.263)$ between the IHCC patients 
Table 2 Prognostic factors for post-operative complications in patients receiving major liver resection before and after PSM

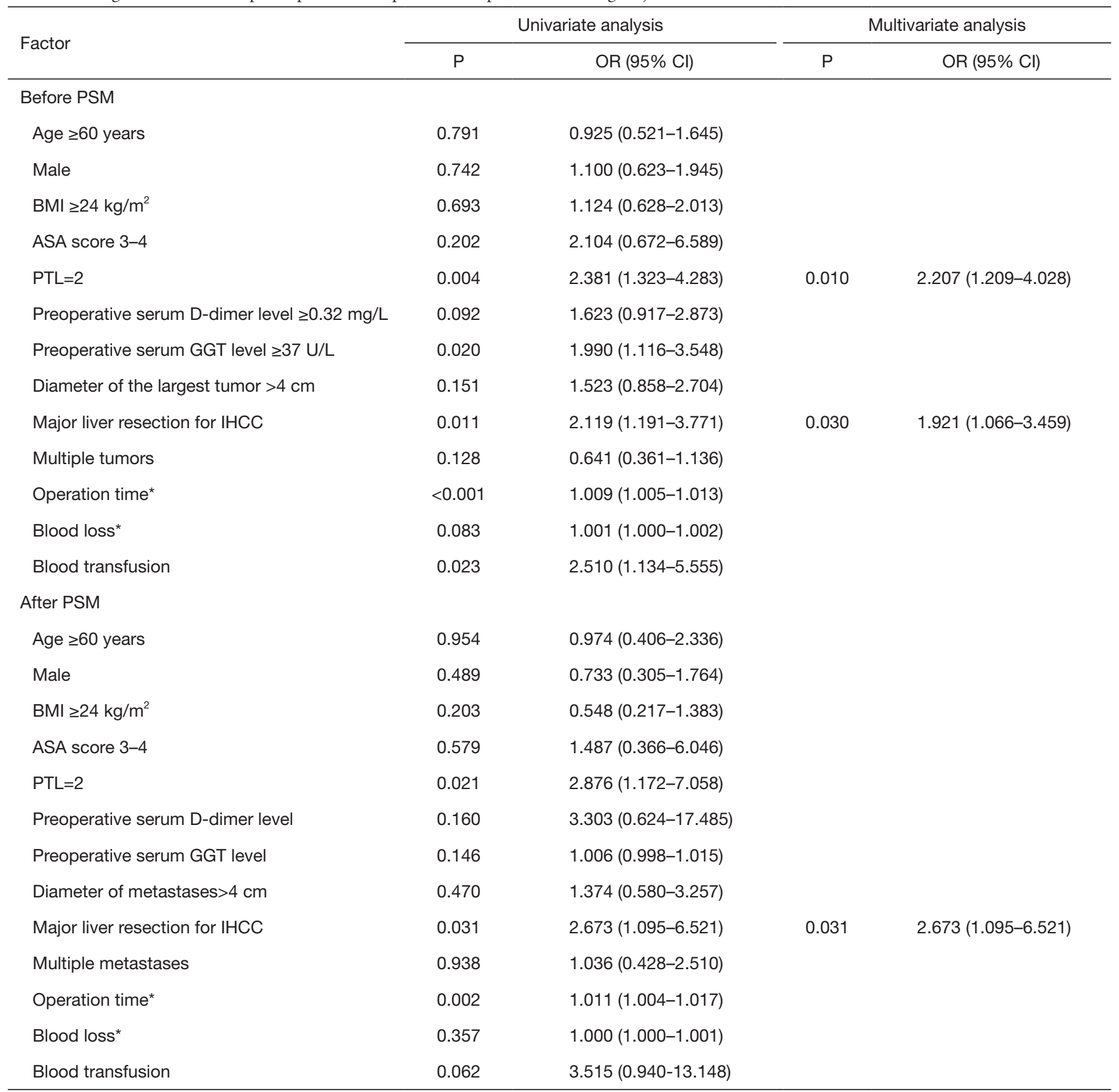

*, operation time and blood loss $\geq$ the median of different surgical methods. PSM, propensity score matching; BMI, body mass index; ASA, American Society of Anesthesiology; PTL, pre-operative treatment and lymphadenectomy; GGT, gamma glutamyl transpeptidase.

and CRLM patients (Table 5).

\section{Discussion}

Our study found that intraoperative outcomes and the incidence of postoperative complications were different between IHCC patients and CRLM patients in different hepatectomy procedures. In the major hepatectomy group, patients with IHCC were more likely to have postoperative complications. In patients with postoperative complications, 
Table 3 Clinicopathological characteristics in patients receiving major liver resection after PSM

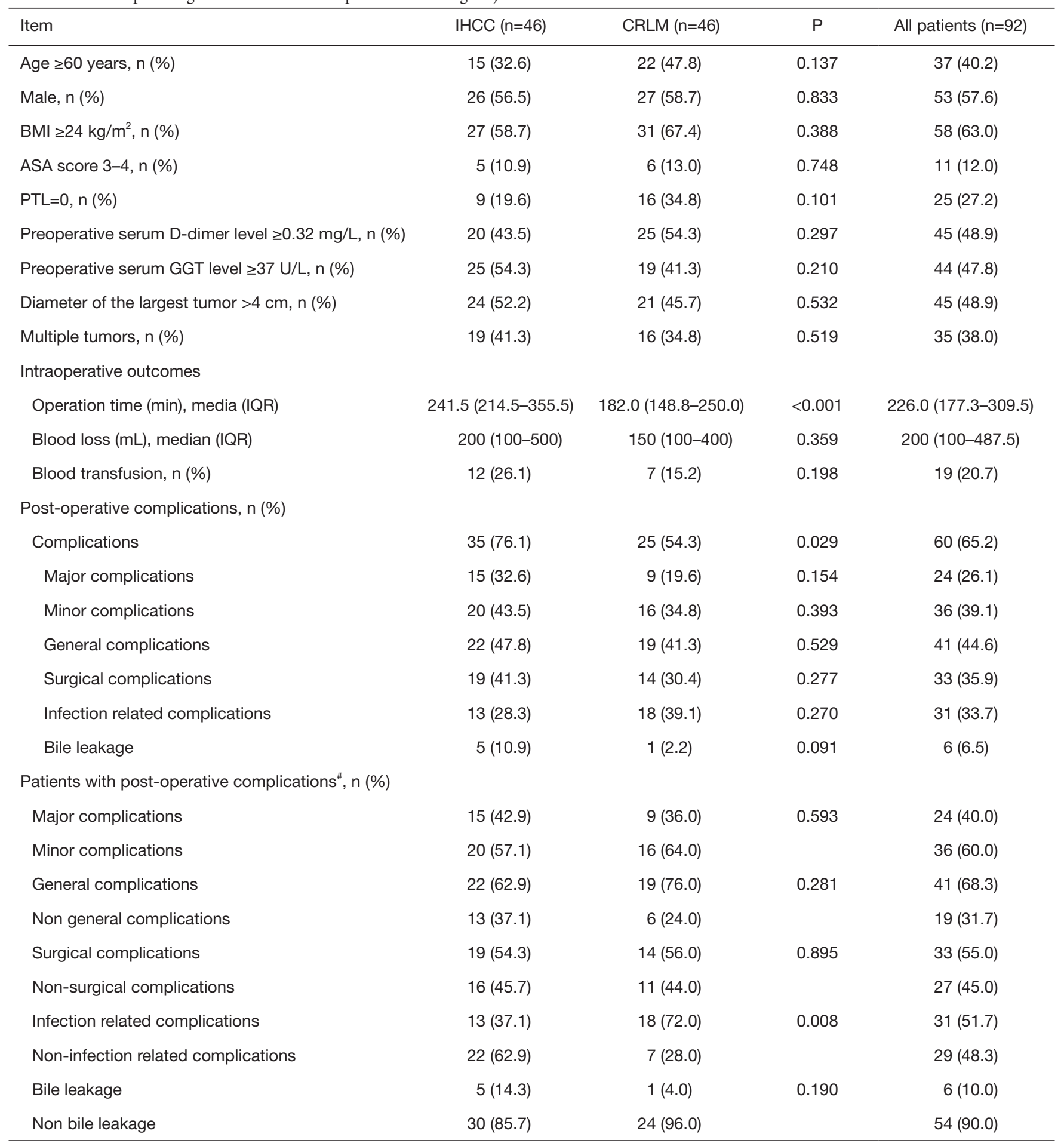

\#, IHCC $(n=35)$, CRLM ( $n=25)$, all patients $(n=60)$. PSM, propensity score matching; BMI, body mass index; ASA, American Society of Anesthesiology; PTL, pre-operative treatment and lymphadenectomy; GGT, gamma glutamyl transpeptidase. 
Table 4 Clinicopathological characteristics in patients receiving minor liver resection before PSM

\begin{tabular}{|c|c|c|c|c|}
\hline Item & $\mathrm{IHCC}(\mathrm{n}=46)$ & CRLM $(n=62)$ & $\mathrm{P}$ & All patients $(n=108)$ \\
\hline Male, $\mathrm{n}(\%)$ & $27(58.7)$ & $38(61.3)$ & 0.785 & $65(60.2)$ \\
\hline $\mathrm{BMI} \geq 24 \mathrm{~kg} / \mathrm{m}^{2}, \mathrm{n}(\%)$ & $26(56.5)$ & $39(62.9)$ & 0.503 & $65(60.2)$ \\
\hline ASA score 3-4, n (\%) & $6(13.0)$ & $2(3.2)$ & 0.054 & $8(7.4)$ \\
\hline Preoperative serum D-dimer level $\geq 0.32 \mathrm{mg} / \mathrm{L}, \mathrm{n}(\%)$ & $20(43.5)$ & $31(50.0)$ & 0.502 & $51(47.2)$ \\
\hline Preoperative serum GGT level $\geq 37$ U/L, n (\%) & $18(39.1)$ & $19(30.6)$ & 0.358 & $37(34.3)$ \\
\hline Diameter of the largest tumor $>4 \mathrm{~cm}, \mathrm{n}(\%)$ & $21(45.7)$ & $9(14.5)$ & $<0.001$ & $30(27.8)$ \\
\hline Multiple tumors, n (\%) & $4(8.7)$ & 8 (12.9) & 0.491 & $12(11.1)$ \\
\hline Blood loss (mL), median (IQR) & $200.0(100.0-300.0)$ & $150.0(95.0-200.0)$ & $<0.001$ & $200(100-300)$ \\
\hline Blood transfusion, $\mathrm{n}(\%)$ & $3(6.5)$ & $0(0.0)$ & 0.041 & $3(2.8)$ \\
\hline \multicolumn{5}{|l|}{ Post-operative complications, n (\%) } \\
\hline Complications & $16(34.8)$ & $18(29.0)$ & 0.525 & $34(31.5)$ \\
\hline Major complications & $5(10.9)$ & $7(11.3)$ & 0.945 & $12(11.1)$ \\
\hline Minor complications & $11(23.9)$ & $11(17.7)$ & 0.431 & $22(20.4)$ \\
\hline General complications & $14(30.4)$ & $15(24.2)$ & 0.469 & $29(26.9)$ \\
\hline Surgical complications & $5(10.9)$ & $7(11.3)$ & 0.945 & $12(11.1)$ \\
\hline General complications & $14(87.5)$ & $15(83.3)$ & 0.732 & $29(85.3)$ \\
\hline Non general complications & $2(12.5)$ & $3(16.7)$ & & $5(14.7)$ \\
\hline Surgical complications & $5(31.3)$ & 7 (38.9) & 0.642 & $12(35.3)$ \\
\hline Non-surgical complications & $11(68.8)$ & $11(61.1)$ & & $22(64.7)$ \\
\hline Infection related complications & $8(50.0)$ & $12(66.7)$ & 0.324 & $20(58.8)$ \\
\hline Non-infection related complications & $8(50.0)$ & $6(33.3)$ & & $14(41.2)$ \\
\hline Bile leakage & $1(6.3)$ & $1(5.6)$ & 0.932 & $2(5.9)$ \\
\hline Non bile leakage & $15(93.8)$ & $17(94.4)$ & & $32(94.1)$ \\
\hline
\end{tabular}

\#, IHCC $(n=16)$, CRLM ( $n=18)$, all patients $(n=34)$. PSM, propensity score matching; BMI, body mass index; ASA, American Society of Anesthesiology; PTL, pre-operative treatment and lymphadenectomy; GGT, gamma glutamyl transpeptidase. 
Table 5 Clinicopathological characteristics in patients receiving minor liver resection after PSM

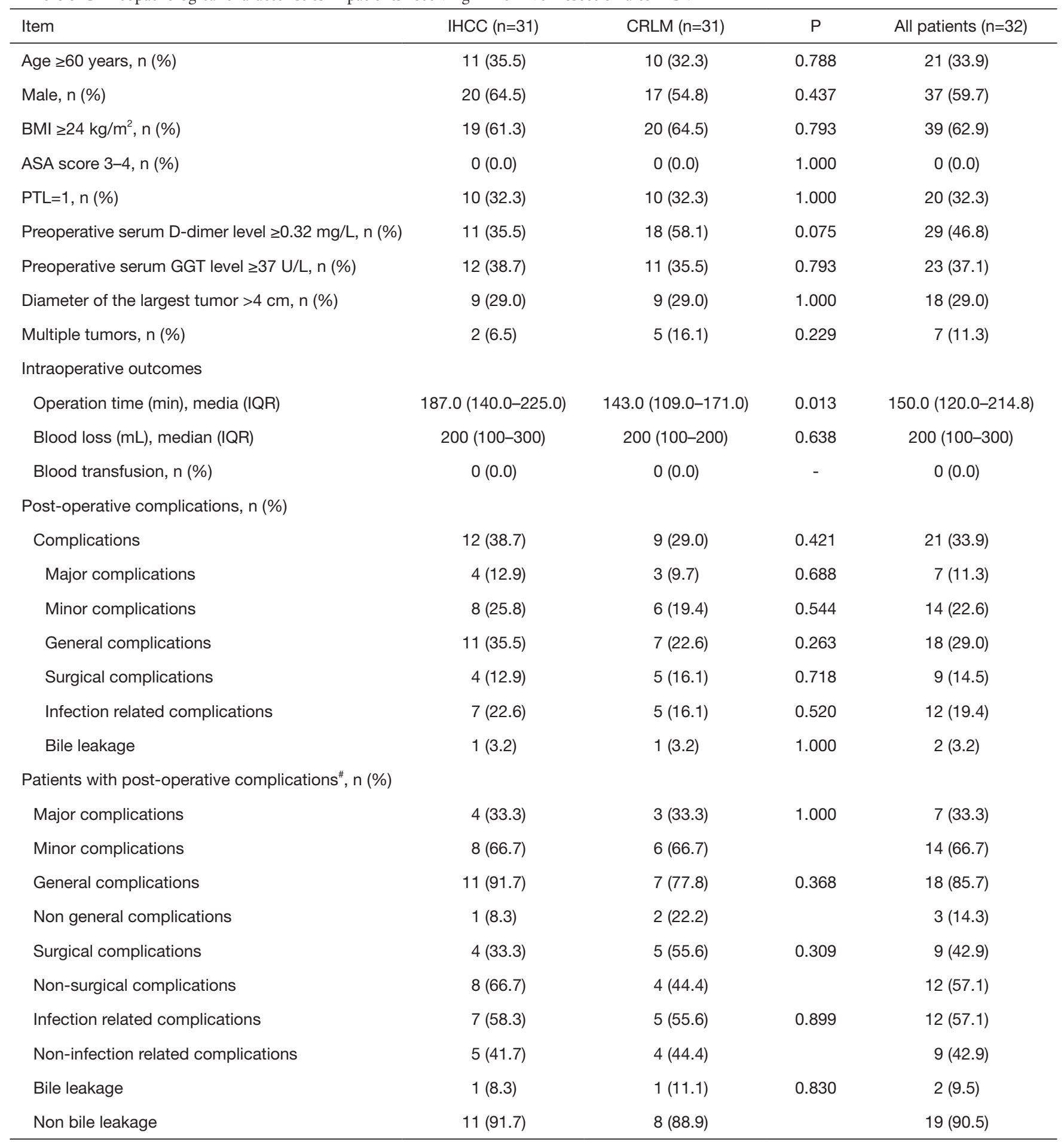

\#, IHCC $(n=12)$, CRLM $(n=9)$, all patients $(n=21)$. PSM, propensity score matching; BMI, body mass index; ASA, American Society of Anesthesiology; PTL, pre-operative treatment and lymphadenectomy; GGT, gamma glutamyl transpeptidase. 
the infection-related complication rate was significantly higher in the CRLM patients than in the IHCC patients. For minor hepatectomy, there was no significant difference in postoperative complications between IHCC and CRLM. The operative time of IHCC patients was longer whatever in the major or minor hepatectomy group.

Early postoperative complications commonly seen in patients with hepatectomy are postoperative bleeding, ascites, infection, and liver failure. Liver failure is the most serious one of them, resulting in postoperative mortality $(21,22)$. The incidence of complications in this study reached $53.9 \%$, including $65.4 \%$ in major and $31.5 \%$ in minor hepatectomy. The most common complication in our study was infection. CRLM patients were more likely to develop infection-related complications during major hepatectomy, which might be related to preoperative adjuvant therapy and secondary surgery. Besides, obesity has been implicated as a risk factor for the development of all-stage colorectal cancer by affecting insulin pathways $(23,24)$. More than $60 \%$ of patients with CRLM had a $\mathrm{BMI} \geq 24 \mathrm{~kg} / \mathrm{m}^{2}$ in this study. And CRLM patients are more likely to have postoperative hyperglycemia (25). Previous studies indicated patients who developed postoperative hyperglycemia after colorectal operations had an increased risk of infection $(26,27)$. Surgical complications, such as postoperative bleeding and deep venous thrombus, were not different between IHCC and CRLM. In patients who underwent hepatectomy, bile leakage remained a common cause of postresectional liver failure (28), with an incidence of $3.4-12.9 \%(29,30)$. However, the morbidity of bile leakage in IHCC patients was not higher than CRLM, though peripheral bile duct dilatation and lymph node dissection were more common in IHCC.

The incidence of postoperative complications between different types of hepatic malignancies was not compared comprehensively in most other centers. In our study, the morbidity of overall complications in the IHCC group was significantly higher the CRLM group. Even after PSM, the rate remained high. And major hepatectomy was a risk factor for the postoperative complication in IHCC group, but not in the CRLM group. Postoperative complications might be not only related to the range of hepatectomy but also the type of hepatic malignancy. In other words, IHCC patients might be inherently at higher operative risk as compared to CRLM patients, possibly due to the biology of the tumor.

Multiple studies have proven that postoperative complications were related to the operation time $(10,31,32)$.
Longer operative time and greater bleeding volume increased the risk of post-hepatectomy morbidity and mortality in both open and laparoscopic hepatectomy $(14,15)$. In this study, the operation time of IHCC was longer than CRLM. Biliary and vascular resections was more common in the surgery of IHCC, which need longer operative time. The location of IHCC always close to the porta hepatis and the retrohepatic inferior vena cava and the tumor size of IHCC was significantly bigger. Another possible reason was routine lymph node dissection for IHCC, while it was not performed routinely for CRLM. Therefore, longer operative time were associated with the characteristics of IHCC.

When patients received major hepatectomy, the morbidity of postoperative complications in IHCC was higher than CRLM. But when they received a minor one, there was no significant difference. It was not surprising because the vascular and biliary injury may be inevitable and the injury can lead to specific postoperative complications such as biliary ischemia or lymphatic leak during portal lymphadenectomy (10). In other studies, age, preoperative liver function Child-Pugh score, range of hepatectomy, and intraoperative bleeding were independent risk factors for postoperative complications of hepatic malignancies (20,33-36).

Our study did have some limitations. First, it was retrospective and single-institutional. Second, there was no other center to validate our conclusion. In the future, we hope there will be more studies to verify the results. Despite these limitations, this study may lead to some comprehensive understanding of these differences. First, considering that in the study the operation for CRLM was secondary surgery, it would take a longer time than the first operation. Both IHCC and CRLM pursued R0 resection, IHCC did not specifically emphasize the extension of the range of hepatectomy compared to CRLM. Previous studies did not clarify the cases of simultaneous and heterogeneous CRLM, nor did they compare the cases of preoperative adjuvant therapy. We took this into account and only heterogeneous CRLM were selected. Second, the rate of complication could also be related to lymph node dissection for IHCC (37) or preoperative neoadjuvant treatment for CRLM (38). Preoperative neoadjuvant chemotherapy was common for CRLM, but only a small percentage of patients with IHCC received preoperative neoadjuvant chemotherapy. Hence no matter which of the two was matched in PSM, the size of the other group would be too small. In this case, the combination of the status of PTL was established to analyze the postoperative complications. 
In conclusion, our study confirmed that major hepatectomy for IHCC led to significantly higher morbidity of complications than CRLM patients. Nowadays, the oncological resection of tumors remains the gold standard for therapeutic intents, so more attention should be paid to improve surgical techniques and management of hepatic malignancies especially in the setting of IHCC, which will lead to a favorable outcome after surgery.

\section{Acknowledgments}

Funding: This study was supported by the State Key Project on Infection Diseases of China (Grant No. 2017ZX10201021-007-003), the National The capital health research and development of special (Grant No. 2018-1-4021), the National Natural Science Foundation of China (Grant No. 81672461, 81972311), CAMS Innovation Fund for Medical Sciences(CIFMS)(Grant no.201712M-4-002), the Non-profit Central Research Institution Fund of Chinese Academy of Medical Sciences (Grant No. 2019PT310026) and Sanming Project of Medicine in Shenzhen (Grant No. SZSM202011010).

\section{Footnote}

Reporting Checklist: The authors have completed the STROBE reporting checklist. Available at https://dx.doi. org/10.21037/tcr-21-553

Data Sharing Statement: Available at https://dx.doi. org/10.21037/tcr-21-553

Peer Review File: Available at https://dx.doi.org/10.21037/ tcr-21-553

Conflicts of Interest: All authors have completed the ICMJE uniform disclosure form (available at https://dx.doi. org/10.21037/tcr-21-553). The authors have no conflicts of interest to declare.

Ethical Statement: The authors are accountable for all aspects of the work in ensuring that questions related to the accuracy or integrity of any part of the work are appropriately investigated and resolved. The study was conducted in accordance with the Declaration of Helsinki (as revised in 2013). The study was approved by the Institutional Ethics Committee of Cancer Hospital Chinese Academy of Medical Sciences (ID NCC2019C-016).
Informed consent was taken from all the patients.

Open Access Statement: This is an Open Access article distributed in accordance with the Creative Commons Attribution-NonCommercial-NoDerivs 4.0 International License (CC BY-NC-ND 4.0), which permits the noncommercial replication and distribution of the article with the strict proviso that no changes or edits are made and the original work is properly cited (including links to both the formal publication through the relevant DOI and the license). See: https://creativecommons.org/licenses/by-nc-nd/4.0/.

\section{References}

1. Bridgewater J, Galle PR, Khan SA, et al. Guidelines for the diagnosis and management of intrahepatic cholangiocarcinoma. J Hepatol 2014;60:1268-89.

2. Yoshino T, Arnold D, Taniguchi H, et al. Pan-Asian adapted ESMO consensus guidelines for the management of patients with metastatic colorectal cancer: a JSMOESMO initiative endorsed by CSCO, KACO, MOS, SSO and TOS. Ann Oncol 2018;29:44-70.

3. Chun YS, Javle M. Systemic and Adjuvant Therapies for Intrahepatic Cholangiocarcinoma. Cancer Control 2017;24:1073274817729241.

4. van Amerongen MJ, van der Stok EP, Fütterer JJ, et al. Short term and long term results of patients with colorectal liver metastases undergoing surgery with or without radiofrequency ablation. Eur J Surg Oncol 2016;42:523-30.

5. Norén A, Eriksson HG, Olsson LI. Selection for surgery and survival of synchronous colorectal liver metastases; a nationwide study. Eur J Cancer 2016;53:105-14.

6. Zhang XF, Bagante F, Chen Q, et al. Perioperative and long-term outcome of intrahepatic cholangiocarcinoma involving the hepatic hilus after curative-intent resection: comparison with peripheral intrahepatic cholangiocarcinoma and hilar cholangiocarcinoma. Surgery 2018;163:1114-20.

7. El-Diwany R, Pawlik TM, Ejaz A. Intrahepatic Cholangiocarcinoma. Surg Oncol Clin N Am 2019;28:587-99.

8. Ardito F, Panettieri E, Vellone M, et al. The impact of $\mathrm{R} 1$ resection for colorectal liver metastases on local recurrence and overall survival in the era of modern chemotherapy: An analysis of 1,428 resection areas. Surgery 2019;165:712-20.

9. Cillo U, Fondevila C, Donadon M, et al. Surgery for 
cholangiocarcinoma. Liver Int 2019;39 Suppl 1:143-55.

10. Doussot A, Fuks D, Regimbeau JM, et al. Major hepatectomy for intrahepatic cholangiocarcinoma or colorectal liver metastases. Are we talking about the same story? Eur J Surg Oncol 2019;45:2353-9.

11. Ubink I, Jongen JMJ, Nijkamp MW, et al. Surgical and Oncologic Outcomes After Major Liver Surgery and Extended Hemihepatectomy for Colorectal Liver Metastases. Clin Colorectal Cancer 2016;15:e193-8.

12. Dupré A, Lefranc A, Buc E, et al. Use of bioresorbable membranes to reduce abdominal and perihepatic adhesions in 2-stage hepatectomy of liver metastases from colorectal cancer: results of a prospective, randomized controlled phase II trial. Ann Surg 2013;258:30-6.

13. Dorcaratto D, Mazzinari G, Fernandez M, et al. Impact of Postoperative Complications on Survival and Recurrence After Resection of Colorectal Liver Metastases: Systematic Review and Meta-analysis. Ann Surg 2019;270:1018-27.

14. Le Roy B, Gelli M, Pittau G, et al. Neoadjuvant chemotherapy for initially unresectable intrahepatic cholangiocarcinoma. Br J Surg 2018;105:839-47.

15. Zhang XF, Bagante F, Chakedis J, et al. Perioperative and Long-Term Outcome for Intrahepatic Cholangiocarcinoma: Impact of Major Versus Minor Hepatectomy. J Gastrointest Surg 2017;21:1841-50.

16. Memeo R, de Blasi V, Adam R, et al. Postoperative Infectious Complications Impact Long-Term Survival in Patients Who Underwent Hepatectomies for Colorectal Liver Metastases: a Propensity Score Matching Analysis. J Gastrointest Surg 2018;22:2045-54.

17. De Blasi V, Memeo R, Adam R, et al. Major Hepatectomy for Colorectal Liver Metastases in Patients Aged Over 80: A Propensity Score Matching Analysis. Dig Surg 2018;35:333-41.

18. Truant S, Séquier C, Leteurtre E, et al. Tumour biology of colorectal liver metastasis is a more important factor in survival than surgical margin clearance in the era of modern chemotherapy regimens. HPB (Oxford) 2015;17:176-84.

19. Laurent C, Adam JP, Denost Q, et al. Significance of R1 Resection for Advanced Colorectal Liver Metastases in the Era of Modern Effective Chemotherapy. World J Surg 2016;40:1191-9.

20. Kingham TP, Correa-Gallego C, D'Angelica MI, et al. Hepatic parenchymal preservation surgery: decreasing morbidity and mortality rates in 4,152 resections for malignancy. J Am Coll Surg 2015;220:471-9.

21. Gilg S, Sandström P, Rizell M, et al. The impact of post- hepatectomy liver failure on mortality: a population-based study. Scand J Gastroenterol 2018;53:1335-9.

22. Kubota K, Aoki T, Kumamaru H, et al. Use of the National Clinical Database to evaluate the association between preoperative liver function and postoperative complications among patients undergoing hepatectomy. J Hepatobiliary Pancreat Sci 2019;26:331-40.

23. Pathak S, Pandanaboyana S, Daniels I, et al. Obesity and colorectal liver metastases: Mechanisms and management. Surg Oncol 2016;25:246-51.

24. Rasool S, Kadla SA, Rasool V, et al. A comparative overview of general risk factors associated with the incidence of colorectal cancer. Tumour Biol 2013;34:2469-76.

25. Margonis GA, Amini N, Sasaki K, et al. Perioperative Hyperglycemia and Postoperative Outcomes in Patients Undergoing Resection of Colorectal Liver Metastases. J Gastrointest Surg 2017;21:228-37.

26. Ramos M, Khalpey Z, Lipsitz S, et al. Relationship of perioperative hyperglycemia and postoperative infections in patients who undergo general and vascular surgery. Ann Surg 2008;248:585-91.

27. Kwon S, Thompson R, Dellinger $\mathrm{P}$, et al. Importance of perioperative glycemic control in general surgery: a report from the Surgical Care and Outcomes Assessment Program. Ann Surg 2013;257:8-14.

28. van Mierlo KM, Schaap FG, Dejong CH, et al. Liver resection for cancer: New developments in prediction, prevention and management of postresectional liver failure. J Hepatol 2016;65:1217-31.

29. Andreatos N, Amini N, Gani F, et al. Albumin-Bilirubin Score: Predicting Short-Term Outcomes Including Bile Leak and Post-hepatectomy Liver Failure Following Hepatic Resection. J Gastrointest Surg 2017;21:238-48.

30. Martin AN, Narayanan S, Turrentine FE, et al. Clinical Factors and Postoperative Impact of Bile Leak After Liver Resection. J Gastrointest Surg 2018;22:661-7.

31. Shin TH, Naples R, French JC, et al. Effect modification of resident autonomy and seniority on perioperative outcomes in laparoscopic cholecystectomy. Surg Endosc 2021;35:3387-97.

32. Song KB, Hong S, Kim HJ, et al. Predictive Factors Associated with Complications after Laparoscopic Distal Pancreatectomy. J Clin Med 2020;9:2766.

33. Shen P, Fino N, Levine EA, et al. Bile duct surgery in the treatment of hepatobiliary and gallbladder malignancies: effects of hepatic and vascular resection on outcomes. HPB (Oxford) 2015;17:1066-73. 
34. Gómez-Gavara C, Doussot A, Lim C, et al. Impact of intraoperative blood transfusion on short and long term outcomes after curative hepatectomy for intrahepatic cholangiocarcinoma: a propensity score matching analysis by the AFC-IHCC study group. HPB (Oxford) 2017;19:411-20.

35. Cloyd JM, Mizuno T, Kawaguchi Y, et al. Comprehensive Complication Index Validates Improved Outcomes Over Time Despite Increased Complexity in 3707 Consecutive Hepatectomies. Ann Surg 2020;271:724-31.

36. Dokmak S, Ftériche FS, Borscheid R, et al. 2012 Liver

Cite this article as: Chen Q, Zheng Y, Chen J, Zhou J, Zhao J, Bi X, Li Z, Huang Z, Zhang Y, Wu J, Wang L, Zhao H, Cai J. Differences of intraoperative outcomes and postoperative complications between intrahepatic cholangiocarcinoma and colorectal liver metastasis in different surgical methods. Transl Cancer Res 2021;10(9):4020-4032. doi: 10.21037/tcr-21-553 resections in the 21st century: we are far from zero mortality. HPB (Oxford) 2013;15:908-15.

37. Miyata T, Yamashita YI, Yamao T, et al. Prognostic impacts of postoperative complications in patients with intrahepatic cholangiocarcinoma after curative operations. Int J Clin Oncol 2017;22:526-32.

38. Lubezky N, Winograd E, Papoulas M, et al. Perioperative complications after neoadjuvant chemotherapy with and without bevacizumab for colorectal liver metastases. J Gastrointest Surg 2013;17:527-32. 